NISSUNA UMANA INVESTIGAZIONE SI PUO DIMANDARE VERA SCIENZIA S'ESSA NON PASSA PER LE MATEMATICHE DIMOSTRAZIONI LEONARDO DA VINCI

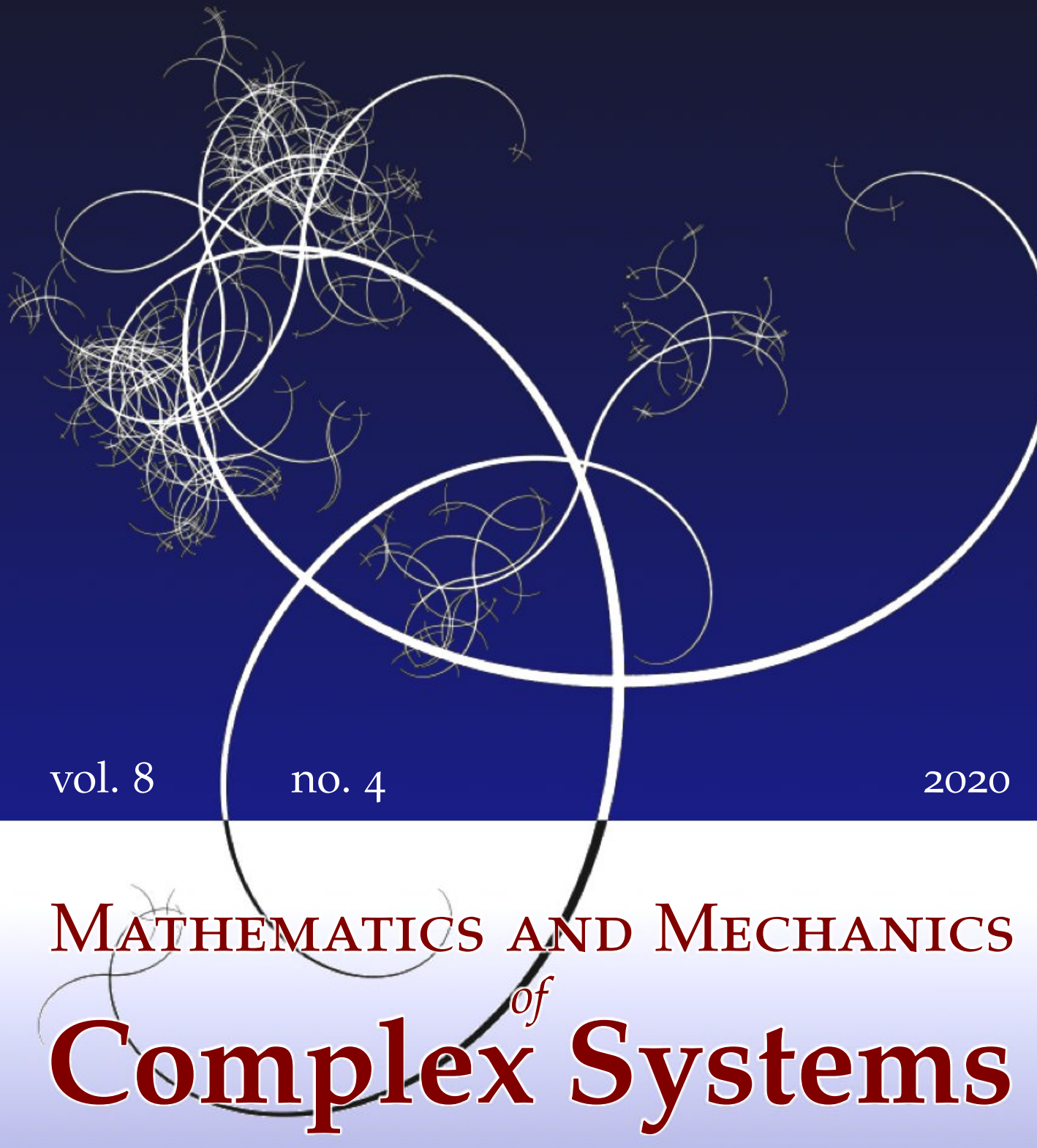

PAUL STEINMANN

ANALYTICAL MECHANICS ALLOWS NOVEL VISTAS ON MATHEMATICAL EPIDEMIC DYNAMICS MODELING 


\title{
ANALYTICAL MECHANICS ALLOWS NOVEL VISTAS ON MATHEMATICAL EPIDEMIC DYNAMICS MODELING
}

\author{
PAUL STEINMANN
}

\begin{abstract}
This contribution aims to shed light on mathematical epidemic dynamics modeling from the viewpoint of analytical mechanics. To set the stage, it recasts the basic SIR model of mathematical epidemic dynamics in an analytical mechanics setting. Thereby, it considers two possible reparametrizations of the basic SIR model: one rescales time, while the other transforms the coordinates, i.e., the independent variables. In both cases, Hamilton's equations in terms of a suited Hamiltonian as well as Hamilton's principle in terms of a suited Lagrangian are considered in minimal and extended phase and state space coordinates, respectively. The corresponding Legendre transformations relating the various options for the Hamiltonians and Lagrangians are detailed. Ultimately, this contribution expands on a multitude of novel vistas on mathematical epidemic dynamics modeling that emerge from the analytical mechanics viewpoint. As a result, it is believed that interesting and relevant new research avenues open up when exploiting in depth the analogies between analytical mechanics and mathematical epidemic dynamics modeling.
\end{abstract}

\section{Motivation}

The global COVID-19 pandemic, with alleged outbreak by the end of 2019 in Wuhan, China [Lu et al. 2020] - despite its devastating implications for health, economy, and society - has in particular challenged modeling and simulation of mathematical epidemic dynamics. Political decision makers around the globe seek (or should seek) advice from scientists such as virologists, biologists, clinicians, economists, and sociologists as well as modelers from different fields. Especially the latter are in the position to virtually simulate various scenarios based on wellfounded assumptions in order to provide support and guidance for the difficult and momentous decisions of politicians, e.g., on lockdown measures and stepwise exit strategies thereof. Thus, the critical importance of modeling is clearly appreciated, and indeed, mathematical epidemic dynamics modeling is a well-established and mature field.

\section{Communicated by Francesco dell'Isola.}

MSC2020: 70K99.

Keywords: epidemics modeling, analytical mechanics. 
Traditional mathematical modeling of epidemic dynamics is rooted in the concept of susceptible, infected, and recovered (SIR) compartments as originally proposed in [Kermack and McKendrick 1927; 1932; 1933; 1937; 1939]. Various modifications extend the classical SIR model to account for further compartments such as, e.g., deceased (SIRD model), exposed (SEIR model), and quarantined (SIQRD model), among many other sophisticated options [Hethcote 2000; Diekmann et al. 2013]. Classical SIR-type compartment-based models are coupled ordinary differential equations (ODEs). Extending the ODE-based SIR-type modeling approach to integro-differential equations (IDEs) allows one to also consider the detailed course of the disease, e.g., delay due to incubation time and the infectious period [Keimer and Pflug 2020]. SIR-type models describe the temporal spread of infectious diseases for integral populations, thereby, however, neglecting the interconnectedness of spatially distributed geographic areas. Recently proposed multiple compartment models, e.g., the mcSIR model in [Seroussi et al. 2019], also allow consideration of the geographical spread of potentially multiple infectious virus strains within the population and its potentially multiple subgroups (e.g., age groups). Spatial network models, e.g., [Balcan et al. 2009; 2010; Pastor-Satorras et al. 2015], for example based on the SEIR model at each network node can qualitatively simulate the outbreak dynamics of infectious diseases and the impact of travel restrictions in geographical areas at the global (macro) scale such as China and the USA [Peirlinck et al. 2020] or Europe [Linka et al. 2020]. However, due to its stochastic nature and strong impact of socio-economic factors, modeling epidemic dynamics within geographical areas at the local (micro) scale requires the use of another modeling paradigm, i.e., rule-driven, agent-based models [Rahmandad and Sterman 2008]. Agent-based models allow for example studying the effect of various lockdown exit strategies on local geographical entities with only a comparatively small number of individuals (agents); see, e.g., [German et al. 2020]. Regardless of the modeling approach taken, quantitative predictions of epidemic dynamics remain challenging and critically require careful identification of model parameters from reliable databases; see, e.g., [Kergaßner et al. 2020b].

Given this mature state of affairs, why is a novel, alternative view on mathematical epidemic dynamics modeling justified at all?

The answer is as follows: to date, modelers of complex mechanical systems and behavior have developed a versatile and extremely successful toolset, including sophisticated analytical and in particular efficient and accurate computational methods. Examples are techniques to master severe nonlinearities and couplings with nonmechanical fields, a multitude of multiscale and homogenization modeling approaches as well as incorporating uncertainty quantification into modeling and simulation. Mathematical epidemic dynamics modeling can undoubtedly benefit 
largely from this accumulated expertise! In summary, it is therefore believed that first translating epidemic dynamics models into an analytical mechanics setting (related steps towards this aim may be found, e.g., in [Militaru and Munteanu 2013; Ionescu et al. 2015; Seroussi et al. 2019]) and then, secondly, exploiting the analogy between the two approaches while utilizing the full toolset of mechanical modeling can provide novel vistas and unprecedented opportunities. The present contribution aims to sketch out a few of these perspectives and to encourage the mechanics community to offer its strong modeling expertise to possibly and hopefully help further improve epidemic dynamics modeling.

\section{Basic SIR model}

Classical modeling of epidemics dynamics is rooted in the concept of susceptible, infected, and recovered (SIR) compartments as originally proposed in [Kermack and McKendrick 1927; 1932; 1933; 1937; 1939]. The basic compartment-based SIR model is the set of two coupled ordinary differential equations (ODEs)

$$
\left[\begin{array}{l}
I^{\bullet} \\
S^{\bullet}
\end{array}\right]=\left[\begin{array}{c}
\beta S I-\gamma I \\
-\beta S I
\end{array}\right]
$$

Here, $I$ and $S$ denote the stock of individuals in the infected and the susceptible compartments, respectively, normalized by the size of the entire population. The notation for the derivative of a quantity with respect to ordinary time $t$ is

$$
\{\cdot\}^{\bullet}:=\mathrm{d}_{t}\{\cdot\} .
$$

The parameters $\beta$ and $\gamma$ are the infection and the recovery rate, respectively, with their ratio defining the basic reproduction number $R_{0}:=\beta / \gamma$. Note finally that the stock of individuals in the recovered compartment follows from the constraint $S+I+R=1$; thus, the evolution equation $R^{\bullet}=-\left[S^{\bullet}+I^{\bullet}\right]$ is tacitly suppressed in our presentation.

\section{Time reparametrized SIR model}

In order to recast the basic SIR model into a format more amenable to the analytical mechanics setting, it is proposed, as a first option, to rescale time as

$$
\tau:=\int_{0}^{t} S I \mathrm{~d} \bar{t} \quad \text { with } \quad \mathrm{d} \tau / \mathrm{d} t=S I .
$$

The notation for the derivative of a quantity with respect to rescaled time $\tau$ is

$$
\{\cdot\}^{\circ}:=\mathrm{d}_{\tau}\{\cdot\} \text {. }
$$


Consequently, the derivatives with respect to rescaled and ordinary time are related via

$$
\{\cdot\}^{\circ}=\{\cdot\}^{\bullet} /[S I] .
$$

As a result, the basic SIR model is eventually reparametrized in terms of rescaled time as

$$
\left[\begin{array}{l}
I^{\circ} \\
S^{\circ}
\end{array}\right]=\boldsymbol{F} \quad \text { with } \quad \boldsymbol{F}:=\left[\begin{array}{c}
\beta-\gamma / S \\
-\beta
\end{array}\right]=:\left[\begin{array}{l}
V \\
F
\end{array}\right] .
$$

In the time reparametrized SIR model the right-hand side is abbreviated as the forcing term $\boldsymbol{F}$, i.e., as the column matrix consisting of the time reparametrized rate of infection $V$ and force of infection $F$ (a common terminology from mathematical epidemiology already establishing a semantic analogy to mechanics). Computing $I^{\circ \circ}=\left[\gamma / S^{2}\right] S^{\circ}$ from $(6)_{1}$ and eliminating $S^{\circ}$ with the help of $(6)_{2}$ renders $I^{\circ \circ}=$ $-\beta \gamma / S^{2}$. Resorting finally again to $(6)_{1}$, i.e., expressing $S\left(I^{\circ}\right)=\gamma /\left[\beta-I^{\circ}\right]$, allows formulating the time reparametrized SIR model as a single nonlinear ODE

$$
I^{\circ \circ}=-R_{0}\left[\beta-I^{\circ}\right]^{2}
$$

exclusively in the stock of individuals in the infected compartment and with the basic reproduction number and the infection rate as parameters.

3.1. Hamiltonian in minimal phase space coordinates. The minimal phase space coordinates collectively assembled in the column matrix $\boldsymbol{Z} \in \mathbb{R}^{2}$, i.e., the generalized coordinate $Q$ defined as the stock of individuals in the infected compartment and the generalized momentum $P$ defined as the stock of individuals in the susceptible compartment, span the two-dimensional phase space $\mathbb{P}$; thus,

$$
\mathbb{P}:=\left\{\boldsymbol{Z}:=\left[\begin{array}{l}
Q \\
P
\end{array}\right]:=\left[\begin{array}{l}
I \\
S
\end{array}\right]\right\} .
$$

The Hamiltonian $H(\boldsymbol{Z})$ in minimal phase space coordinates, which eventually results in the time reparametrized SIR model from (6), is then identified as

$$
H(\boldsymbol{Z}):=\beta[I+S]-\gamma \ln S .
$$

Indeed, the corresponding Hamilton equations deliver a reformulation of the result in (6), i.e.,

$$
\left[\begin{array}{l}
I^{\circ} \\
S^{\circ}
\end{array}\right]=\left[\begin{array}{rr}
0 & 1 \\
-1 & 0
\end{array}\right]\left[\begin{array}{c}
\beta \\
\beta-\gamma / S
\end{array}\right]
$$

Symbolic notation clearly reveals the Hamiltonian structure of the time reparametrized SIR model

$$
\boldsymbol{Z}^{\circ}=\boldsymbol{J} \cdot \partial_{\boldsymbol{Z}} H(\boldsymbol{Z}) .
$$


Thereby, the skew-symmetric $\boldsymbol{J} \in \mathbb{R}^{2} \times \mathbb{R}^{2}$ denotes the so-called symplectic matrix in $\mathbb{P}$

$$
\boldsymbol{J}:=\left[\begin{array}{rr}
0 & 1 \\
-1 & 0
\end{array}\right]
$$

with $\boldsymbol{J}^{t}=-\boldsymbol{J}$ and $\boldsymbol{J}^{2}=-\boldsymbol{I}$, where $\boldsymbol{I} \in \mathbb{R}^{2} \times \mathbb{R}^{2}$ is the common unit matrix in $\mathbb{P}$. The Hamiltonian structure in terms of the skew-symmetric $\boldsymbol{J}$ clearly identifies the (autonomous) Hamiltonian $H(\boldsymbol{Z})$ in minimal phase space coordinates as first integral, i.e., as a conserved quantity under the flow implied by the Hamilton equations, since

$$
H^{\circ}=\partial_{\mathbf{Z}} H \cdot \boldsymbol{Z}^{\circ}=\partial_{\mathbf{Z}} H \cdot \boldsymbol{J} \cdot \partial_{\mathbf{Z}} H=0 .
$$

The gradient of the Hamiltonian $H(\boldsymbol{Z})$ with respect to the minimal phase space coordinates, abbreviated in the sequel as $\boldsymbol{G}(\boldsymbol{Z}) \in \mathbb{R}^{2}$, is computed as

$$
\partial_{\boldsymbol{Z}} H(\boldsymbol{Z})=\left[\begin{array}{c}
\beta \\
\beta-\gamma / S
\end{array}\right]=: \boldsymbol{G}(\boldsymbol{Z}) .
$$

Taken together, the time reparametrized SIR model obeys Hamiltonian structure and identifies the relation between the gradient $\boldsymbol{G}(\boldsymbol{Z}) \in \mathbb{R}^{2}$ of the Hamiltonian $H(\boldsymbol{Z})$ in minimal phase space coordinates and the forcing term $\boldsymbol{F}(\boldsymbol{Z}) \in \mathbb{R}^{2}$ as

$$
Z^{\circ}=\boldsymbol{J} \cdot \boldsymbol{G}(\boldsymbol{Z})=\boldsymbol{F}(\boldsymbol{Z}) .
$$

Exchanging the time derivative to the one with respect to ordinary time $t$ destroys the clean Hamiltonian structure in terms of a constant symplectic matrix; i.e.,

$$
\boldsymbol{Z} \cdot=[S I] \boldsymbol{J} \cdot \boldsymbol{G}(\boldsymbol{Z})=[S I] \boldsymbol{F}(\boldsymbol{Z}) .
$$

One may, of course, reinterpret this result as a Hamiltonian structure with nonconstant, coordinate-dependent symplectic matrix $[S I] J$ on a nonflat manifold.

3.2. Lagrangian in minimal state space coordinate. The minimal state space coordinate, i.e., the generalized coordinate $Q$ defined as the stock of individuals in the infected compartment, spans the one-dimensional state space $\mathbb{S}$; thus,

$$
\mathbb{S}:=\{Q:=I\} .
$$

Then a Legendre transformation of the Hamiltonian in minimal phase space coordinates defines the corresponding Lagrangian

$$
L\left(I, I^{\circ}\right):=\sup _{S}\left\{S I^{\circ}-H(\boldsymbol{Z})\right\} .
$$

The supremum condition identifies $I^{\circ}$ with the derivative $\partial_{S} H(\boldsymbol{Z})$ of the Hamiltonian in minimal phase space coordinates from (9) with respect to the generalized 
momentum, and renders

$$
I^{\circ} \doteq \beta-\gamma / S
$$

Resolving the above supremum condition for $S$ in terms of $I^{\circ}$ delivers

$$
S\left(I^{\circ}\right)=\gamma /\left[\beta-I^{\circ}\right]
$$

Then, with $S\left(I^{\circ}\right) I^{\circ}=\gamma I^{\circ} /\left[\beta-I^{\circ}\right]$ and $H\left(I, S\left(I^{\circ}\right)\right)=\beta\left[I+\gamma /\left[\beta-I^{\circ}\right]\right]-$ $\gamma \ln \left(\gamma /\left[\beta-I^{\circ}\right]\right)$, the Lagrangian in minimal state space coordinate follows eventually as

$$
L\left(I, I^{\circ}\right)=-\beta I-\gamma+\gamma \ln \left(\gamma /\left[\beta-I^{\circ}\right]\right) .
$$

Based on the Lagrangian in minimal state space coordinates, Hamilton's principle results in the stationarity condition

$$
\left[\frac{\partial L}{\partial I^{\circ}}\right]^{\circ}=\frac{\partial L}{\partial I} .
$$

Thus, with $\partial_{I^{\circ}} L=\gamma /\left[\beta-I^{\circ}\right] \rightarrow\left[\partial_{I^{\circ}} L\right]^{\circ}=\gamma I^{\circ \circ} /\left[\beta-I^{\circ}\right]^{2}$ and $\partial_{I} L=-\beta$, the Euler-Lagrange equation corresponding to the Lagrangian in minimal state space coordinates reads

$$
I^{\circ \circ}+R_{0}\left[\beta-I^{\circ}\right]^{2}=0 .
$$

Clearly, the Euler-Lagrange equation in minimal state space coordinates coincides with the single, nonlinear ODE formulation of the time reparametrized SIR model in (7).

3.3. Lagrangian in extended state space coordinates. Alternatively, extended state space coordinates collectively assembled in the column matrix $\boldsymbol{Q} \in \mathbb{R}^{2}$, i.e., the generalized coordinates jointly defined as the stock of individuals in the infected and susceptible compartments, span the two-dimensional state space $\overline{\mathbb{S}}$; thus,

$$
\overline{\mathbb{S}}:=\left\{\boldsymbol{Q}:=\left[\begin{array}{l}
I \\
S
\end{array}\right]\right\}
$$

Then the Lagrangian $\bar{L}\left(\boldsymbol{Q}, \boldsymbol{Q}^{\circ}\right)$ in extended state space coordinates, which eventually results in the time reparametrized SIR model from (6), is determined as

$$
\bar{L}\left(\boldsymbol{Q}, \boldsymbol{Q}^{\circ}\right):=\frac{1}{2} \boldsymbol{Q} \cdot \boldsymbol{J}^{t} \cdot \boldsymbol{Q}^{\circ}-H(\boldsymbol{Q}) .
$$

Here, the Legendre transformation term $\boldsymbol{Q} \cdot \boldsymbol{J}^{t} \cdot \boldsymbol{Q}^{\circ}=\boldsymbol{Q}^{\circ} \cdot \boldsymbol{J} \cdot \boldsymbol{Q}$ expands as the skew-symmetric form

$$
\boldsymbol{Q} \cdot \boldsymbol{J}^{t} \cdot \boldsymbol{Q}^{\circ}=S I^{\circ}-I S^{\circ}
$$


whereas the Hamiltonian $H(\boldsymbol{Q})$, corresponding to (9), is now parametrized in terms of the extended state space coordinates $\boldsymbol{Q}$ with

$$
H(Q):=\beta[I+S]-\gamma \ln S .
$$

As a result, the Lagrangian in extended state space coordinates then renders the stationarity conditions of the corresponding Hamilton principle as

$$
\left[\frac{\partial \bar{L}}{\partial \boldsymbol{Q}^{\circ}}\right]^{\circ}=\frac{\partial \bar{L}}{\partial \boldsymbol{Q}}
$$

With $\partial \boldsymbol{Q}^{\circ} \bar{L}=\boldsymbol{Q} \cdot \boldsymbol{J}^{t} / 2$ and $\partial_{\boldsymbol{Q}} \bar{L}=\boldsymbol{Q}^{\circ} \cdot \boldsymbol{J} / 2-\boldsymbol{G}(\boldsymbol{Q})$, the Euler-Lagrange equations thus follow as

$$
\frac{1}{2} \boldsymbol{Q}^{\circ} \cdot \boldsymbol{J}^{t}=\frac{1}{2} \boldsymbol{Q}^{\circ} \cdot \boldsymbol{J}-\boldsymbol{G}(\boldsymbol{Q}) .
$$

Unfolding the compact symbolic notation, these expand concretely into

$$
\frac{1}{2}\left[\begin{array}{r}
S^{\circ} \\
-I^{\circ}
\end{array}\right]=\frac{1}{2}\left[\begin{array}{r}
-S^{\circ} \\
I^{\circ}
\end{array}\right]-\left[\begin{array}{c}
\beta \\
\beta-\gamma / S
\end{array}\right] .
$$

The Euler-Lagrange equations in (29) are next reformulated by recalling that due to the skew-symmetry of the symplectic matrix, $\boldsymbol{Q}^{\circ} \cdot \boldsymbol{J}=-\boldsymbol{Q}^{\circ} \cdot \boldsymbol{J}^{t}$ and $\boldsymbol{Q}^{\circ} \cdot \boldsymbol{J}^{t}=\boldsymbol{J} \cdot \boldsymbol{Q}^{\circ}$ hold; thus,

$$
\boldsymbol{J} \cdot \boldsymbol{Q}^{\circ}=-\boldsymbol{G}(\boldsymbol{Q})
$$

Finally, with $\boldsymbol{J}^{2}=-\boldsymbol{I}$, the reformulated Euler-Lagrange equations in (31) are modified into

$$
Q^{\circ}=J \cdot G(Q)=F(Q)
$$

Obviously, this format recovers the relation between the gradient $G(\boldsymbol{Q}) \in \mathbb{R}^{2}$ of the Hamiltonian $H(\boldsymbol{Q})$ (in extended state space coordinates) and the forcing term $\boldsymbol{F}(\boldsymbol{Q}) \in \mathbb{R}^{2}$ already established previously in (15). Likewise, exchanging the time derivative to the one with respect to ordinary time $t$

$$
\boldsymbol{Q}^{\bullet}=[S I] \boldsymbol{J} \cdot \boldsymbol{G}(\boldsymbol{Q})=[S I] \boldsymbol{F}(\boldsymbol{Q})
$$

results again in a formulation with nonconstant, coordinate-dependent symplectic matrix $[S I] J$ on a nonflat manifold.

3.4. Hamiltonian in extended phase space coordinates. For academic curiosity it is also interesting to consider extended phase space coordinates $\boldsymbol{Q}$, i.e., the generalized coordinates jointly defined as the stock of individuals in the infected and susceptible compartments, and $\boldsymbol{P}$, i.e., heretofore undefined generalized momenta, 
collectively assembled in the column matrix $\bar{Z} \in \mathbb{R}^{4}$, to span the four-dimensional state space $\overline{\mathbb{P}}$; thus,

$$
\overline{\mathbb{P}}:=\left\{\overline{\boldsymbol{Z}}:=\left[\begin{array}{l}
\boldsymbol{Q} \\
\boldsymbol{P}
\end{array}\right] \text { with } \boldsymbol{Q}:=\left[\begin{array}{l}
I \\
S
\end{array}\right] \text { and } \boldsymbol{P}:=\left[\begin{array}{c}
\Upsilon \\
\Sigma
\end{array}\right]\right\} .
$$

A Legendre transformation of the Lagrangian in extended state space coordinates from (25) defines the associated Hamiltonian

$$
\bar{H}(\boldsymbol{Q}, \boldsymbol{P})=\sup _{\boldsymbol{Q}^{\circ}}\left\{\boldsymbol{P} \cdot \boldsymbol{Q}^{\circ}-\bar{L}\left(\boldsymbol{Q}, \boldsymbol{Q}^{\circ}\right)\right\} .
$$

The corresponding supremum condition identifies $\boldsymbol{P}$ with $\partial \boldsymbol{Q}^{\circ} \bar{L}\left(\boldsymbol{Q}, \boldsymbol{Q}^{\circ}\right)$, the derivative of the Lagrangian $\bar{L}\left(\boldsymbol{Q}, \boldsymbol{Q}^{\circ}\right)$ in extended state space coordinates from (25) with respect to the time reparametrized rate of the generalized coordinates $\boldsymbol{Q}^{\circ}$, and renders

$$
\frac{\partial \bar{L}}{\partial \boldsymbol{Q}^{\circ}}=\frac{1}{2} \boldsymbol{Q} \cdot \boldsymbol{J}^{t}=: \boldsymbol{P} .
$$

As a result, $\boldsymbol{P}$ does not depend on $\boldsymbol{Q}^{\circ}$, thus identifying the Lagrangian $\bar{L}\left(\boldsymbol{Q}, \boldsymbol{Q}^{\circ}\right)$ in (25) as degenerate in the sense of Dirac's generalized Hamiltonian dynamics [Dirac 1950; 1958]. Consequently, with $\boldsymbol{Q} \cdot \boldsymbol{J}^{t}=\boldsymbol{J} \cdot \boldsymbol{Q}$ and $\boldsymbol{J}^{2}=-\boldsymbol{I}$, an additional constraint for the extended phase space coordinates emerges:

$$
\boldsymbol{C}(\boldsymbol{Q}, \boldsymbol{P})=\boldsymbol{Q}+2 \boldsymbol{J} \cdot \boldsymbol{P} \doteq \mathbf{0} .
$$

The Hamiltonian $\bar{H}(\boldsymbol{Q}, \boldsymbol{P})$ in extended phase space coordinates thus follows from Legendre transformation by incorporating the constraint via the Lagrange multiplier $\boldsymbol{\Lambda}$; i.e.,

$$
\bar{H}(\boldsymbol{Q}, \boldsymbol{P})=\frac{1}{2} \boldsymbol{Q} \cdot \boldsymbol{J}^{t} \cdot \boldsymbol{Q}^{\circ}-\bar{L}\left(\boldsymbol{Q}, \boldsymbol{Q}^{\circ}\right)+\boldsymbol{\Lambda} \cdot \boldsymbol{C}(\boldsymbol{Q}, \boldsymbol{P}) .
$$

Taking into account the explicit form of the Lagrangian $\bar{L}\left(\boldsymbol{Q}, \boldsymbol{Q}^{\circ}\right)$ in extended state space coordinates from (25) then renders the explicit representation of the Hamiltonian $\bar{H}(\boldsymbol{Q}, \boldsymbol{P})$ in extended phase space coordinates:

$$
\bar{H}(\boldsymbol{Q}, \boldsymbol{P})=H(\boldsymbol{Q})+\boldsymbol{\Lambda} \cdot \boldsymbol{C}(\boldsymbol{Q}, \boldsymbol{P}) .
$$

Invoking $\partial_{Q} \boldsymbol{C}(\boldsymbol{Q}, \boldsymbol{P})=\boldsymbol{I}$ and $\partial_{\boldsymbol{P}} \boldsymbol{C}(\boldsymbol{Q}, \boldsymbol{P})=2 \boldsymbol{J}$, Hamilton's equations based on the Hamiltonian $\bar{H}(\boldsymbol{Q}, \boldsymbol{P})$ in extended phase space coordinates from (39) result in

$$
\left[\begin{array}{l}
\boldsymbol{Q}^{\circ} \\
\boldsymbol{P}^{\circ}
\end{array}\right]=\left[\begin{array}{rr}
0 & \boldsymbol{I} \\
-\boldsymbol{I} & 0
\end{array}\right]\left[\begin{array}{l}
\partial_{\boldsymbol{Q}} \bar{H} \\
\partial_{\boldsymbol{P}} \bar{H}
\end{array}\right]=\left[\begin{array}{rr}
0 & \boldsymbol{I} \\
-\boldsymbol{I} & 0
\end{array}\right]\left[\begin{array}{c}
\boldsymbol{G}(\boldsymbol{Q})+\boldsymbol{\Lambda} \\
2 \boldsymbol{\Lambda} \cdot \boldsymbol{J}
\end{array}\right] .
$$

The unknown Lagrange multiplier $\boldsymbol{\Lambda}$ is determined from the consistency condition for the constraint

$$
\boldsymbol{C}^{\circ}\left(\boldsymbol{Q}^{\circ}, \boldsymbol{P}^{\circ}\right)=\boldsymbol{Q}^{\circ}+2 \boldsymbol{J} \cdot \boldsymbol{P}^{\circ} \doteq \mathbf{0},
$$


which, upon introducing Hamilton's equations $\boldsymbol{Q}^{\circ}=2 \boldsymbol{\Lambda} \cdot \boldsymbol{J}$ and $\boldsymbol{P}^{\circ}=-[\boldsymbol{G}(\boldsymbol{Q})+\boldsymbol{\Lambda}]$, results in

$$
2 \boldsymbol{\Lambda} \cdot \boldsymbol{J}-2 \boldsymbol{J} \cdot[\boldsymbol{G}(\boldsymbol{Q})+\boldsymbol{\Lambda}] \doteq \mathbf{0} .
$$

The solution of the consistency condition then renders the explicit representation for the Lagrange multiplier

$$
\boldsymbol{\Lambda}=-\frac{1}{2} \boldsymbol{G}(\boldsymbol{Q}) .
$$

Using again Hamilton's equation $\boldsymbol{Q}^{\circ}=2 \boldsymbol{\Lambda} \cdot \boldsymbol{J}$ and exploiting the skew-symmetry of the symplectic matrix, i.e., $\boldsymbol{\Lambda} \cdot \boldsymbol{J}=-\boldsymbol{J} \cdot \boldsymbol{\Lambda}$, recovers once more the already previously established relation between the gradient $\boldsymbol{G}(\boldsymbol{Q}) \in \mathbb{R}^{2}$ of the Hamiltonian $H(\boldsymbol{Q})$ (in extended state space coordinates) and the forcing term $\boldsymbol{F}(\boldsymbol{Q}) \in \mathbb{R}^{2}$ :

$$
Q^{\circ}=\boldsymbol{J} \cdot \boldsymbol{G}(\boldsymbol{Q})=\boldsymbol{F}(\boldsymbol{Q}) \text {. }
$$

Finally, using Hamilton's equation $\boldsymbol{P}^{\circ}=-[\boldsymbol{G}(\boldsymbol{Q})+\boldsymbol{\Lambda}]$ identifies eventually the time reparametrized rate of the heretofore unknown generalized momenta as

$$
\boldsymbol{P}^{\circ}=-\frac{1}{2} \boldsymbol{G}(\boldsymbol{Q})=\frac{1}{2} \boldsymbol{J} \cdot \boldsymbol{F}(\boldsymbol{Q}) .
$$

The last equality follows from $\boldsymbol{J}^{2}=-\boldsymbol{I}$ and the previous relation $\boldsymbol{J} \cdot \boldsymbol{G}(\boldsymbol{Q})=\boldsymbol{F}(\boldsymbol{Q})$.

\section{Coordinate reparametrized SIR model}

Alternatively, and again aiming to recast the basic SIR model into an analytical mechanics format, it is proposed, as a second option, to logarithmically transform the coordinates (or rather the independent variables) as

$$
I \mapsto i:=\ln I \quad \text { and } \quad S \mapsto s:=\ln S .
$$

As a result, the basic SIR model, reparametrized in terms of logarithmically transformed coordinates, however still in terms of derivatives with respect to ordinary time $t$, now reads

$$
\left[\begin{array}{l}
i^{\bullet} \\
s^{\bullet}
\end{array}\right]=f \quad \text { with } \quad f:=\left[\begin{array}{c}
\beta S(s)-\gamma \\
-\beta I(i)
\end{array}\right]=:\left[\begin{array}{l}
v \\
f
\end{array}\right] .
$$

Note that in this reparametrization, the stocks of individuals in the infected and susceptible compartments are considered as dependent functions of the logarithmically transformed coordinates

$$
I(i):=\exp i \text { and } S(s):=\exp s .
$$

In the coordinate reparametrized SIR model the right-hand side is abbreviated as the forcing term $f$, i.e., as the column matrix consisting of the coordinate reparametrized rate of infection $v$ and force of infection $f$. Computing $i^{\bullet}=\beta S(s) s^{\bullet}$ 
from $(47)_{1}$ and eliminating $s^{\bullet}$ with the help of $(47)_{2}$ renders $i^{\bullet \bullet}=-\beta^{2} S(s) I(i)$. Resorting finally again to $(47)_{1}$, i.e., expressing $S(s)=[i \cdot \gamma] / \beta$, allows formulating the coordinate reparametrized SIR model as a single nonlinear ODE

$$
i^{\bullet \bullet}=-\beta I(i)\left[i^{\bullet}+\gamma\right]
$$

exclusively in the logarithmic stock of individuals in the infected compartment.

4.1. Hamiltonian in minimal phase space coordinates. The minimal (logarithmic) phase space coordinates collectively assembled in the column matrix $z \in \mathbb{R}^{2}$, i.e., the generalized coordinate $q$ defined as the logarithmic stock of individuals in the infected compartment and the generalized momentum $p$ defined as the logarithmic stock of individuals in the susceptible compartment, span the two-dimensional phase space $p$; thus,

$$
\mathrm{p}:=\left\{z:=\left[\begin{array}{l}
q \\
p
\end{array}\right]:=\left[\begin{array}{l}
i \\
s
\end{array}\right]\right\}
$$

Next, the Hamiltonian $h(z)$ in minimal (logarithmic) phase space coordinates, which eventually results in the coordinate reparametrized SIR model from (47), is identified as

$$
h(z):=\beta[I(i)+S(s)]-\gamma s .
$$

As a result, the corresponding Hamilton equations deliver a reformulation of (47), i.e.,

$$
\left[\begin{array}{l}
i^{\bullet} \\
s^{\bullet}
\end{array}\right]=\left[\begin{array}{rr}
0 & 1 \\
-1 & 0
\end{array}\right]\left[\begin{array}{c}
\beta I(i) \\
\beta S(s)-\gamma
\end{array}\right]
$$

Symbolic notation showcases clearly the Hamiltonian structure of the (logarithmic) coordinate reparametrized SIR model

$$
\boldsymbol{z}^{\bullet}=\boldsymbol{j} \cdot \partial_{z} h(\boldsymbol{z})
$$

Here, the skew-symmetric $\boldsymbol{j} \in \mathbb{R}^{2} \times \mathbb{R}^{2}$ denotes the appropriate symplectic matrix in $p$

$$
\boldsymbol{j}:=\left[\begin{array}{rr}
0 & 1 \\
-1 & 0
\end{array}\right]
$$

with $\boldsymbol{j}^{t}=-\boldsymbol{j}$ and $\boldsymbol{j}^{2}=-\boldsymbol{i}$, where $\boldsymbol{i} \in \mathbb{R}^{2} \times \mathbb{R}^{2}$ is the common unit matrix in $p$. The Hamiltonian structure in terms of the skew-symmetric $\boldsymbol{j}$ clearly identifies the (autonomous) Hamiltonian $h(z)$ in minimal (logarithmic) phase space coordinates as first integral:

$$
h^{\bullet}=\partial_{z} h \cdot z^{\bullet}=\partial_{z} h \cdot \boldsymbol{j} \cdot \partial_{z} h=0
$$


The gradient of the Hamiltonian $h(z)$ with respect to the minimal (logarithmic) phase space coordinates, abbreviated in the sequel as $g(z) \in \mathbb{R}^{2}$, is computed as

$$
\partial_{z} h(z)=\left[\begin{array}{c}
\beta I(i) \\
\beta S(s)-\gamma
\end{array}\right]=: \boldsymbol{g}(\boldsymbol{z}) .
$$

Summarizing, the coordinate reparametrized SIR model obeys Hamiltonian structure and identifies the relation between the gradient $g(z) \in \mathbb{R}^{2}$ of the Hamiltonian $h(z)$ in minimal (logarithmic) phase space coordinates and the forcing term $\boldsymbol{f}(\boldsymbol{z}) \in \mathbb{R}^{2}$ as

$$
z^{\bullet}=j \cdot g(z)=f(z)
$$

It is emphasized that despite the logarithmic nature of the reparametrized coordinates, here, as a benefit, the Hamiltonian structure involves the constant, coordinateindependent symplectic matrix $\boldsymbol{j}$ of a flat manifold as well as derivatives with respect to ordinary time $t$.

4.2. Lagrangian in minimal state space coordinate. The minimal (logarithmic) state space coordinate, i.e., the generalized coordinate $q$ defined as the logarithmic stock of individuals in the infected compartment, span the one-dimensional state space $s$; thus,

$$
\mathcal{s}:=\{q:=i\}
$$

Legendre transformation of the Hamiltonian in minimal (logarithmic) phase space coordinates defines the corresponding Lagrangian

$$
l\left(i, i^{\bullet}\right)=\sup _{s}\left\{s i^{\bullet}-h(z)\right\} .
$$

Then the supremum condition identifies $i^{\bullet}$ with the derivative $\partial_{s} h(z)$ of the Hamiltonian in minimal (logarithmic) phase space coordinates from (51) with respect to the generalized momentum, and renders

$$
i^{\bullet}=\beta S(s)-\gamma
$$

Resolving the above supremum condition for $s$ in terms of $i^{\bullet}$ delivers

$$
s\left(i^{\bullet}\right)=\ln \left(\left[i^{\bullet}+\gamma\right] / \beta\right) .
$$

Then, with $s\left(i^{\bullet}\right) i^{\bullet}=\ln \left(\left[i^{\bullet}+\gamma\right] / \beta\right) i^{\bullet}$ and $h\left(i, s\left(i^{\bullet}\right)\right)=\beta\left[I(i)+\left[i^{\bullet}+\gamma\right] / \beta\right]-$ $\gamma \ln \left(\left[i^{\bullet}+\gamma\right] / \beta\right)$, the Lagrangian in minimal (logarithmic) state space coordinate follows eventually as

$$
l\left(i, i^{\bullet}\right)=-\beta I(i)-\left[i^{\bullet}+\gamma\right]\left[1-\ln \left(\left[i^{\bullet}+\gamma\right] / \beta\right)\right] .
$$


Based on the Lagrangian in minimal (logarithmic) state space coordinates, Hamilton's principle results in the stationarity condition

$$
\left[\frac{\partial l}{\partial i}\right]^{\bullet}=\frac{\partial l}{\partial i} .
$$

Thus, with $\partial_{i} \bullet l=-\left[1-\ln \left(\left[i^{\bullet}+\gamma\right] / \beta\right)\right]+\beta \rightarrow\left[\partial_{i} \bullet l\right]^{\bullet}=i^{\bullet \bullet} /\left[i^{\bullet}+\gamma\right]$ and $\partial_{i} l=$ $-\beta I(i)$, the Euler-Lagrange equation corresponding to the Lagrangian in minimal (logarithmic) state space coordinates reads

$$
i^{\bullet}+\beta I(i)[i \cdot \gamma]=0 .
$$

Obviously, the Euler-Lagrange equation in minimal (logarithmic) state space coordinates coincides with the single, nonlinear ODE formulation of the coordinate reparametrized SIR model in (49).

4.3. Lagrangian in extended state space coordinates. Alternatively, extended (logarithmic) state space coordinates collectively assembled in the column matrix $\boldsymbol{q} \in$ $\mathbb{R}^{2}$, i.e., the generalized coordinates jointly defined as the logarithmic stock of individuals in the infected and susceptible compartments, span the two-dimensional state space $\overline{\mathrm{s}}$; thus,

$$
\bar{s}:=\left\{\boldsymbol{q}:=\left[\begin{array}{l}
i \\
s
\end{array}\right]\right\} .
$$

The Lagrangian $\bar{l}\left(\boldsymbol{q}, \boldsymbol{q}^{\bullet}\right)$ in extended (logarithmic) state space coordinates, which eventually results in the coordinate reparametrized SIR model from (47), reads

$$
\bar{l}\left(\boldsymbol{q}, \boldsymbol{q}^{\bullet}\right):=\frac{1}{2} \boldsymbol{q} \cdot \boldsymbol{j}^{t} \cdot \boldsymbol{q}^{\bullet}-h(\boldsymbol{q}) .
$$

Here, the Legendre transformation term $\boldsymbol{q} \cdot \boldsymbol{j}^{t} \cdot \boldsymbol{q}^{\bullet}=\boldsymbol{q}^{\bullet} \cdot \boldsymbol{j} \cdot \boldsymbol{q}$ expands as the skewsymmetric form

$$
\boldsymbol{q} \cdot \boldsymbol{j}^{t} \cdot \boldsymbol{q}^{\bullet}=s \boldsymbol{i}^{\bullet}-i s^{\bullet}
$$

The Hamiltonian $h(\boldsymbol{q})$, corresponding to (51), is now parametrized in terms of the extended (logarithmic) state space coordinates $\boldsymbol{q}$ as

$$
h(\boldsymbol{q}):=\beta[I(i)+S(s)]-\gamma s .
$$

In extended (logarithmic) state space coordinates the Lagrangian then renders the stationarity conditions of the corresponding Hamilton principle

$$
\left[\frac{\partial \bar{l}}{\partial \boldsymbol{q}^{\bullet}}\right]^{\bullet}=\frac{\partial \bar{l}}{\partial \boldsymbol{q}}
$$

With $\partial_{\boldsymbol{q}} \cdot \bar{l}=\boldsymbol{q} \cdot \boldsymbol{j}^{t} / 2$ and $\partial_{\boldsymbol{q}} \bar{l}=\boldsymbol{q}^{\bullet} \cdot \boldsymbol{j} / 2-\boldsymbol{g}(\boldsymbol{q})$, the Euler-Lagrange equations thus follow as

$$
\frac{1}{2} \boldsymbol{q}^{\bullet} \cdot \boldsymbol{j}^{t}=\frac{1}{2} \boldsymbol{q}^{\bullet} \cdot \boldsymbol{j}-\boldsymbol{g}(\boldsymbol{q})
$$


and concretely unfold into

$$
\frac{1}{2}\left[\begin{array}{r}
s^{\bullet} \\
-i^{\bullet}
\end{array}\right]=\frac{1}{2}\left[\begin{array}{r}
-s^{\bullet} \\
i^{\bullet}
\end{array}\right]-\left[\begin{array}{c}
\beta I(i) \\
\beta S(s)-\gamma
\end{array}\right] .
$$

Subsequently, the Euler-Lagrange equations in (70) are reformulated by noting that $\boldsymbol{q}^{\bullet} \cdot \boldsymbol{j}=-\boldsymbol{q}^{\bullet} \cdot \boldsymbol{j}^{t}$ and $\boldsymbol{q}^{\bullet} \cdot \boldsymbol{j}^{t}=\boldsymbol{j} \cdot \boldsymbol{q}^{\bullet} ;$ thus,

$$
j \cdot q^{\bullet}=-g(q) .
$$

Finally, with $\boldsymbol{j}^{2}=-\boldsymbol{i}$, the Euler-Lagrange equations from (72) reformulate as

$$
q^{\bullet}=j \cdot g(q)=f(q)
$$

This format recovers the relation between the gradient $\boldsymbol{g}(\boldsymbol{q}) \in \mathbb{R}^{2}$ of the Hamiltonian $h(\boldsymbol{q})$ (in extended (logarithmic) state space coordinates) and the forcing term $\boldsymbol{f}(\boldsymbol{q}) \in \mathbb{R}^{2}$ already established previously in (57).

4.4. Hamiltonian in extended phase space coordinates. For completeness we also consider extended (logarithmic) phase space coordinates $\boldsymbol{q}$, i.e., the generalized coordinates jointly defined as the logarithmic stock of individuals in the infected and susceptible compartments, and $\boldsymbol{p}$, i.e., heretofore undefined generalized momenta, collectively assembled in the column matrix $\bar{z} \in \mathbb{R}^{4}$, to span the four-dimensional state space $\bar{p}$; thus,

$$
\overline{\mathbb{p}}:=\left\{\bar{z}:=\left[\begin{array}{l}
\boldsymbol{q} \\
\boldsymbol{p}
\end{array}\right] \text { with } \boldsymbol{q}:=\left[\begin{array}{l}
i \\
s
\end{array}\right] \text { and } \boldsymbol{p}:=\left[\begin{array}{l}
v \\
\sigma
\end{array}\right]\right\} .
$$

A Legendre transformation of the Lagrangian in extended (logarithmic) state space coordinates from (66) defines the associated Hamiltonian

$$
\bar{h}(\boldsymbol{q}, \boldsymbol{p})=\sup _{\boldsymbol{q}^{\bullet}}\left\{\boldsymbol{p} \cdot \boldsymbol{q}^{\bullet}-\bar{l}\left(\boldsymbol{q}, \boldsymbol{q}^{\bullet}\right)\right\} .
$$

The Legendre transformation identifies $\boldsymbol{p}$ with the derivative $\partial_{\boldsymbol{q}} \cdot \bar{l}\left(\boldsymbol{q}, \boldsymbol{q}^{\bullet}\right)$ of the Lagrangian $\bar{l}\left(\boldsymbol{q}, \boldsymbol{q}^{\bullet}\right)$ in extended (logarithmic) state space coordinates from (66) with respect to the rate of the generalized coordinates $\boldsymbol{q}^{\bullet}$, and renders

$$
\frac{\partial \bar{l}}{\partial \boldsymbol{q}}=\frac{1}{2} \boldsymbol{q} \cdot \boldsymbol{j}^{t}=: \boldsymbol{p}
$$

Thus, $\boldsymbol{p}$ does not depend on $\boldsymbol{q}^{\bullet}$, consequently identifying the Lagrangian $\bar{l}\left(\boldsymbol{q}, \boldsymbol{q}^{\bullet}\right)$ in (66) as degenerate [Dirac 1950; 1958]. Consequently, with $\boldsymbol{q} \cdot \boldsymbol{j}^{t}=\boldsymbol{j} \cdot \boldsymbol{q}$ and $\boldsymbol{j}^{2}=-\boldsymbol{i}$, an additional constraint for the extended (logarithmic) phase space coordinates results:

$$
c(q, p)=q+2 j \cdot p \doteq \mathbf{0}
$$


The Hamiltonian $\bar{h}(\boldsymbol{q}, \boldsymbol{p})$ in extended (logarithmic) phase space coordinates thus follows from Legendre transformation by incorporating the constraint via the Lagrange multiplier $\lambda$; i.e.,

$$
\bar{h}(\boldsymbol{q}, \boldsymbol{p})=\frac{1}{2} \boldsymbol{q} \cdot \boldsymbol{j}^{t} \cdot \boldsymbol{q}^{\bullet}-\bar{l}\left(\boldsymbol{q}, \boldsymbol{q}^{\bullet}\right)+\lambda \cdot \boldsymbol{c}(\boldsymbol{q}, \boldsymbol{p}) .
$$

Taking into account the explicit form of the Lagrangian $\bar{l}\left(\boldsymbol{q}, \boldsymbol{q}^{\bullet}\right)$ in extended (logarithmic) state space coordinates from (66) results in the explicit representation of the Hamiltonian $\bar{h}(\boldsymbol{q}, \boldsymbol{p})$ in extended (logarithmic) phase space coordinates

$$
\bar{h}(\boldsymbol{q}, \boldsymbol{p})=h(\boldsymbol{q})+\lambda \cdot \boldsymbol{c}(\boldsymbol{q}, \boldsymbol{p}) .
$$

Invoking $\partial_{\boldsymbol{q}} \boldsymbol{c}(\boldsymbol{q}, \boldsymbol{p})=\boldsymbol{i}$ and $\partial_{\boldsymbol{p}} \boldsymbol{c}(\boldsymbol{q}, \boldsymbol{p})=2 \boldsymbol{j}$, Hamilton's equations based on the Hamiltonian $\bar{h}(\boldsymbol{q}, \boldsymbol{p})$ in extended (logarithmic) phase space coordinates from (79) result in

$$
\left[\begin{array}{l}
\boldsymbol{q}^{\bullet} \\
\boldsymbol{p}^{\bullet}
\end{array}\right]=\left[\begin{array}{rr}
0 & \boldsymbol{i} \\
-\boldsymbol{i} & 0
\end{array}\right]\left[\begin{array}{l}
\partial_{\boldsymbol{q}} \bar{h} \\
\partial_{\boldsymbol{p}} \bar{h}
\end{array}\right]=\left[\begin{array}{rr}
0 & \boldsymbol{i} \\
-\boldsymbol{i} & 0
\end{array}\right]\left[\begin{array}{c}
\boldsymbol{g}(\boldsymbol{q})+\lambda \\
2 \boldsymbol{\lambda} \cdot \boldsymbol{j}
\end{array}\right]
$$

The unknown Lagrange multiplier $\lambda$ is determined from the consistency condition for the constraint

$$
c^{\bullet}\left(q^{\bullet}, p^{\bullet}\right)=q^{\bullet}+2 j \cdot p^{\bullet}=\mathbf{0}
$$

which, with $\boldsymbol{q}^{\bullet}=2 \lambda \cdot \boldsymbol{j}$ and $\boldsymbol{p}^{\bullet}=-[\boldsymbol{g}(\boldsymbol{q})+\lambda]$, results in

$$
2 \lambda \cdot \boldsymbol{j}-2 \boldsymbol{j} \cdot[\boldsymbol{g}(\boldsymbol{q})+\lambda] \doteq \mathbf{0} .
$$

The solution of the consistency condition then renders the explicit representation for the Lagrange multiplier

$$
\lambda=-\frac{1}{2} g(\boldsymbol{q}) .
$$

Using again Hamilton's equation $\boldsymbol{q}^{\bullet}=2 \boldsymbol{\lambda} \cdot \boldsymbol{j}$ and exploiting $\boldsymbol{\lambda} \cdot \boldsymbol{j}=-\boldsymbol{j} \cdot \boldsymbol{\lambda}$ recovers once more the already previously established relation between the gradient $\boldsymbol{g}(\boldsymbol{q}) \in$ $\mathbb{R}^{2}$ of the Hamiltonian $h(\boldsymbol{q})$ (in extended (logarithmic) state space coordinates) and the forcing term $\boldsymbol{f}(\boldsymbol{q}) \in \mathbb{R}^{2}$ :

$$
q^{\bullet}=j \cdot g(q)=f(q) .
$$

Moreover, using Hamilton's equation $\boldsymbol{p}^{\bullet}=-[\boldsymbol{g}(\boldsymbol{q})+\lambda]$ identifies finally the rate of the heretofore unknown generalized momenta as

$$
p^{\bullet}=-\frac{1}{2} g(q)=\frac{1}{2} \boldsymbol{j} \cdot \boldsymbol{f}(\boldsymbol{q}) .
$$

The last equality follows from $\boldsymbol{j}^{2}=-\boldsymbol{i}$ and the previous relation $\boldsymbol{j} \cdot \boldsymbol{g}(\boldsymbol{q})=\boldsymbol{f}(\boldsymbol{q})$. 


\section{Novel vistas from analytical mechanics}

In order to systematically explore lessons that can be learned from an analytical mechanics viewpoint on mathematical epidemic dynamics modeling, the coordinate reparametrized version of the SIR model, based on the Hamiltonian in phase space, is taken as the point of departure:

$$
z^{\bullet}=j \cdot g(z)
$$

Building on this compact representation, several analytical-mechanics-inspired novel vistas on mathematical epidemic dynamics that promise fruitful research avenues for its modeling are identified in the sequel.

Vista 1. Allowing for nonautonomous, i.e., time-dependent generalized Hamiltonians results in the generalized representation

$$
z^{\bullet}=\boldsymbol{j} \cdot \boldsymbol{g}(z, t) \text {. }
$$

Possible options justifying a nonautonomous Hamiltonian are for example:

(a) Various lockdown measures (cancellation of large events, school closing, contact limitations, etc.) as well as their reversal (exit strategies) at discrete points in time are modeled by time-dependent parameters such as for example the infection and the recovery rates

$$
\beta=\beta(t) \quad \text { and } \quad \gamma=\gamma(t),
$$

thus making the Hamiltonian time-dependent.

(b) Various modifications extend the classical SIR model to account for further compartments such as, e.g., deceased (SIRD model), exposed (SEIR model), and quarantined (SIQRD model), among many other, more sophisticated options [Hethcote 2000; Diekmann et al. 2013]. SIR+ models of these types are then captured by appropriately extending the phase space variables

$$
z:=[I, S, \ldots] \in \mathbb{R}^{2+\cdots}
$$

contributing to the Hamiltonian and its gradient.

(c) Classical SIR-type compartment-based models are coupled ordinary differential equations (ODEs). Extending the ODE-based SIR-type modeling approach to integro-differential equations allows one to also consider, e.g., delay due to incubation time and infectious period [Keimer and Pflug 2020]. For $p$ representing relevant parameters, e.g., continuously distributed risk groups and/or past time, from space $P$, the right-hand side of these read as

$$
\boldsymbol{g}(\boldsymbol{z}, t)=\int_{P} \boldsymbol{\gamma}(z, p, t) \mathrm{d} p
$$

with $\boldsymbol{\gamma}(z, p, t)$ the appropriate $p$-density of $\boldsymbol{g}(z, t)$. 
Vista 2. Allowing for an infinite-dimensional phase space with its coordinates $z=\boldsymbol{z}(\boldsymbol{x}, t) \in \mathbb{R}^{2+\cdots}$ defined as fields in four-dimensional space-time results in the generalized representation

$$
z^{\cdot}=\boldsymbol{j} \cdot \boldsymbol{g}\{\boldsymbol{z}(\boldsymbol{x}), t\} .
$$

Here, the right-hand side is a functional of the phase space coordinates rather than a function. Possible options for an infinite-dimensional phase space are for example:

(a) Gradient-type models, whereby the Hamiltonian depends on the phase space coordinates $\boldsymbol{z}=\boldsymbol{z}(\boldsymbol{x}, t)$ and their higher spatial gradients

$$
\boldsymbol{g}\{\boldsymbol{z}(\boldsymbol{x}), t\}=\delta_{z} h\left(z(\boldsymbol{x}), \nabla_{\boldsymbol{x}} z(\boldsymbol{x}), \ldots, t\right) .
$$

Consequently, the right-hand side follows from the variational derivative of the Hamiltonian, rather than from its gradient. Partial differential equations of reaction-convection-diffusion type describing the spatio-temporal spread of infectious diseases are thus a modeling option [Yamazaki and Wang 2017].

(b) Integral-type models, whereby, similar to peridynamics formulations [Javili et al. 2019], the right-hand side follows from a spatial integration

$$
\boldsymbol{g}\{\boldsymbol{z}(\boldsymbol{x}), t\}=\int_{X} \boldsymbol{\gamma}(z(\boldsymbol{x}), \boldsymbol{x}, t) \mathrm{d} \boldsymbol{x}
$$

over a cut-off domain $X$ (horizon) that covers spatial interaction.

Vista 3. Allowing for a finite-dimensional phase space with its coordinates $\mathbf{z} \in$ $\mathbb{R}^{[2+\cdots] n}$ defined as column matrices results in the generalized representation

$$
\mathbf{z}^{\bullet}=\mathbf{j} \cdot \mathbf{g}(\mathbf{z}, t) .
$$

Possible options for a finite-dimensional phase space are for example:

(a) Partition the entire population into subpopulations, thereby separately considering different age/gender/risk groups (see, e.g., [Pastor-Satorras et al. 2015]):

$$
\mathbf{z}=\left[z_{\text {pop }_{1}}, \ldots, z_{\text {pop }_{\max }}\right] .
$$

(b) Partition into various geographical locations in general network models accounting for the spatio-temporal spread of infectious diseases (see, e.g., [Seroussi et al. 2019]):

$$
\mathbf{z}=\left[z_{\mathrm{loc}_{1}}, \ldots, z_{\mathrm{loc}_{\max }}\right] .
$$

(c) Partition into multiple virus strains providing for generic infectious diseases (see, e.g., [Levy et al. 2018]):

$$
\mathbf{z}=\left[z_{\mathrm{vir}_{1}}, \ldots, \boldsymbol{z}_{\mathrm{vir}_{\max }}\right] .
$$


Capturing the interactions among the various partitions in a network is then reflected by the off-diagonal terms in the Hessian $\mathbf{H}:=\partial_{\mathbf{z z}}^{2} h$ of the Hamiltonian.

Vista 4. For a pandemic such as COVID-19, spatial (geographical) resolution, i.e., resolution of a network, is required at multiple scales: at the global (macro) scale, i.e., for the entire globe; at the medium (meso) scale, i.e., for individual countries; and at the local (micro) scale, i.e., for individual cities/communities. A fully detailed spatial resolution at the local (micro) scale for the entire globe is computationally prohibitive; moreover, most often an overkill degree of detail is also not needed and/or not possible due to the lack of data. However, the spatial resolution shall be adaptive to the quantity of interest, e.g., to study the dynamics of infectious disease spread in a particular city/community, only the integral results of more remote locations on the globe matter. These can be captured by a reduced resolution of the network in those geographically remote locations. This asks for a truly multiscale approach that adaptively zooms in only where needed. Possible options for multiscaling are for example:

(a) Vertical coupling of scales relies on the assumption that the two scales considered are sufficiently separated; see, e.g., [Saeb et al. 2016]. Then the "force" term on the right-hand side can be up-scaled from a subscale model by averaging in the sense of computational homogenization:

$$
\bar{z}^{\bullet}=\langle\mathbf{j} \cdot \mathbf{g}(\mathbf{z}, t ; \bar{z})\rangle .
$$

Here, the sup-scale (indicated by an over-bar) on the left-hand side behaves like an SIR-type model whereas the subscale model at the right-hand side lives either on a finite-dimensional phase space or is represented by a ruledriven, so-called agent-based model. Agent-based models are an alternative modeling paradigm considering only a comparatively small number of individuals (agents). They are capable of capturing the stochastic nature and strong impact of socio-economic factors present at small scales; see, e.g., [German et al. 2020; Rahmandad and Sterman 2008]. The subscale model is driven by the sup-scale phase space coordinates, whereby a proper scale-transition condition defines suited boundary/initial conditions at the subscale.

(b) Horizontal coupling of scales, analogously to the quasicontinuum method [Miller and Tadmor 2002], requires adaptive resolution of the network spacing, here indicated by the sup-script $h$ :

$$
\mathbf{z}_{h}^{\cdot}=\mathbf{j} \cdot \mathbf{g}\left(\mathbf{z}_{h}, t\right) .
$$

Adaptivity requires suited network densification indicators that may follow from a proper error analysis, a topic that is still largely under-investigated for epidemic dynamics models. 
Vista 5. The availability and reliability of recorded data, e.g., regarding the cumulative or daily infection cases, during an epidemic is typically characterized by a large degree of uncertainty, e.g., regarding the infection rates, the degree of immunity, and/or their dark figures. Uncertainty quantification is based on simulations with uncertain data:

$$
\mathbf{z}^{\bullet}=\mathbf{j} \cdot \mathbf{g}(\mathbf{z}(\omega), t)
$$

Thereby, uncertain data is here parametrized in terms of elementary events $\omega$ from which one may repeatedly draw samples to investigate uncertainty propagation throughout our model; see, e.g., [Pivovarov et al. 2018; 2019]. Possible options for the description of uncertainties are for example:

(a) Aleatoric uncertainties require the use of random variables with probability density function (pdf) as a measure of likelihood (e.g., Gaussian pdf in terms of the mean value and standard deviation). Aleatoric uncertainties are stochastic by nature and may not be neglected when the standard deviation is large.

(b) Epistemic uncertainties may be captured by fuzzy variables with possibility density function as a measure of degree of membership (e.g., symmetric triangular membership function in terms of its modal value and support). Epistemic uncertainties reflect a lack of knowledge and, in the case of epidemic dynamics modeling, can be reduced by increasing testing for either infections and/or for antibodies.

Vista 6. The discrete trajectory in time of the phase space variables is algorithmically traced by an integrator of the generic format

$$
\mathbf{z}^{n+1}=\mathbf{z}^{n}+\Delta t \mathbf{j} \cdot \mathbf{g}\left(\mathbf{z}^{n+\alpha}(\omega), t^{n+\alpha}\right) .
$$

Here, subscripts $n+1, n$, and $n+\alpha$ refer to, respectively, the end point, the start point, and an intermediate point of/within a time step of length $\Delta t$. Possible options for time integrators that display different accuracy, stability, and robustness, in particular when integrating nonlinear right-hand sides, are for example:

(a) Runge-Kutta integrators are off-the-shelf algorithms that come in a variety of different flavors (following from the corresponding Butcher tableau) like, e.g., single- and multistage integrators of varying algorithmic accuracy. However, they do not necessarily respect first integrals such as the conservation of the Hamiltonian for autonomous cases and may thus suffer from long-term deterioration of algorithmic stability and robustness. 
(b) Variational integrators are based on a discrete form of the action integral, whereby the integrand of the action integral is given by a discrete Lagrangian

$$
\int_{t^{n}}^{t^{n+1}} l\left(\mathbf{z}^{n+1}, \mathbf{z}^{n}, \Delta t\right) \mathrm{d} t \rightarrow \text { stat. }
$$

The resulting discrete Hamiltonian principle then renders a variational integrator that follows from the discrete action integral being stationary. Variational integrators preserve symmetries (momentum maps) and structure (symplecticity) and are thus characterized by long-term algorithmic accuracy, stability, and robustness; see, e.g., [Lew et al. 2004].

(c) Time-finite-element integrators follow from discretizing the Galerkin (weak) form of the Hamilton equations

$$
\int_{T} \delta \mathbf{z} \cdot\left[\mathbf{z}^{\cdot}-\mathbf{j} \cdot \mathbf{g}\right] \mathrm{d} t=0 \quad \text { for all } \delta \mathbf{z} .
$$

Choosing appropriate Ansatz spaces for the test and trial functions, and suited quadrature rules for approximating the time integrals, render integrators of arbitrary algorithmic accuracy that are also characterized by long-term algorithmic stability and robustness; see, e.g., [Betsch and Steinmann 2000; 2001; 2002; 2005].

Vista 7. The underlying equations governing epidemic dynamics are oftentimes unknown. However, they may be discovered from a data-driven approach [Brunton et al. 2016] if sufficient data is available, a scenario that is typically met for the spatio-temporal spread of infectious diseases. The key idea is then to connect the matrix arrangement of available discrete data points for the rate of the phase space coordinates by a matrix of Ansatz functions, e.g., monomials of the matrix arrangement of available discrete data points for the phase space coordinates, with the matrix arrangement of discrete Ansatz parameters

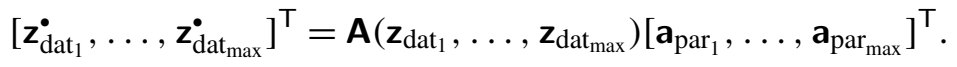

Only few relevant entries in the matrix arrangement of the discrete Ansatz parameters are then determined from sparse regression; consequently the resulting models are denoted as parsimonious and compromise between accuracy and complexity, while avoiding overfitting [Brunton et al. 2016].

Vista 8. Many more modeling approaches inspired by analytical mechanics are conceivable and it is left to the mechanics community to harness those to further improve mathematical epidemic dynamics modeling. 

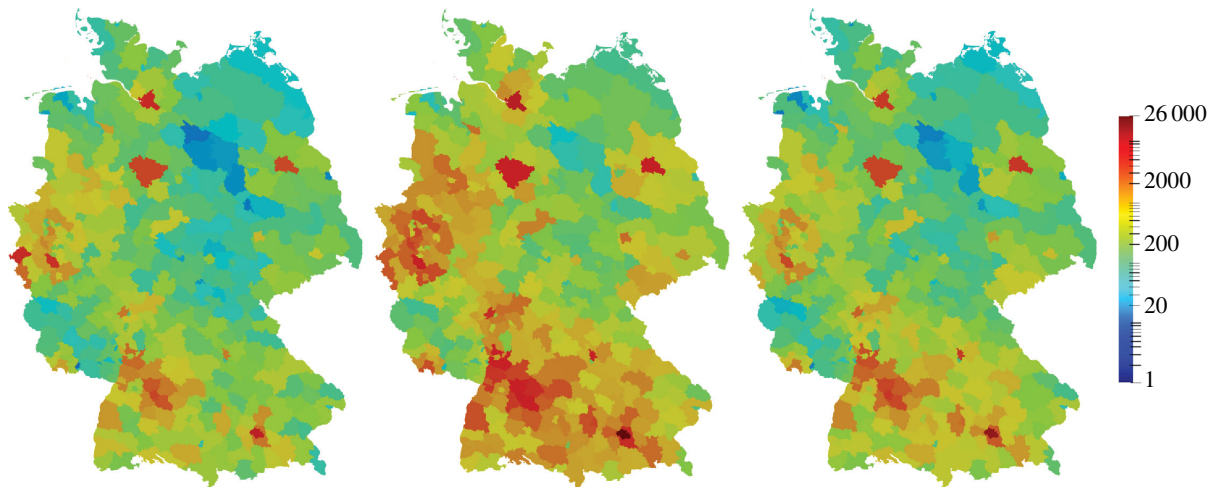

Figure 1. Snapshots of simulated spatio-temporal epidemic spread across Germany with countywise resolution. Colors depict population with COVID-19-specific symptoms on March 13, 2020 (before wave peak), March 27, 2020 (wave peak), and April 10, 2020 (after wave peak). The simulation is based on a spatial network of 401 counties and a convolution-type integro-differential model [Kergaßner et al. 2020a]. The model parameters are calibrated and validated based on extensive data from the Robert Koch Institute (https://corona.rki.de/).

\section{Summary and perspective}

First, this contribution explored options of how to recast the classical SIR model of mathematical epidemic dynamics modeling in the variational setting of analytical mechanics. In particular, it demonstrated that two conceptually entirely different reparametrizations of the basic SIR model, i.e., either by rescaling time or by transforming coordinates (independent variables), severely ease identification of corresponding Hamiltonians and Lagrangians for use within Hamilton's equations and Hamilton's principle. In each case, formulations in either minimal or extended phase and state space coordinates are possible, providing in total eight different modeling options. Interestingly, in minimal phase space coordinates, the stock of individuals in the infected and the susceptible compartments represent the generalized coordinate and the generalized momentum, respectively. In contrast, for extended phase space coordinates, they jointly represent the generalized coordinates, whereas the associated generalized momenta are initially unknown and only follow from exploiting a constraint on the extended phase space coordinates. However, regardless of the particular formulation chosen, from either Hamilton's equations or Hamilton's principle one eventually recovers the original set of coupled ODEs of the SIR model. As a recommendation, logarithmically transforming the coordinates appears more attractive, since derivatives with respect to ordinary time are 
retained for the evolution of the phase space coordinates. As an important perspective, recasting the classical SIR model in one of the eight different modeling options enables the analytical mechanician to employ the full mechanical modeling toolset for a plethora of important extensions. The striking analogy between analytical mechanics and mathematical epidemic dynamics modeling opens up a multitude of fascinating and relevant new research avenues for the progression of the latter. It is thus believed that future exploitation of the Hamiltonian and/or Lagrangian structure of mathematical epidemic dynamics modeling leads to unprecedented insights and options for novel formulations.

As a perspective, Figure 1 showcases (previously unpublished) snapshots of the simulated spatio-temporal epidemic spread across Germany with countywise resolution as an example for the potential of combining, e.g., Vista 1(a)-(c) and Vista 3(b) [Kergaßner et al. 2020a].

\section{Acknowledgement}

I gratefully acknowledge the support of my coauthors for [Kergaßner et al. 2020a; 2020b] in providing the data and simulations for Figure 1.

\section{References}

[Balcan et al. 2009] D. Balcan, V. Colizza, B. Gonçalves, H. Hu, J. J. Ramasco, and A. Vespignani, "Multiscale mobility networks and the spatial spreading of infectious diseases", P. Natl. Acad. Sci. USA 106:51 (2009), 21484-21489.

[Balcan et al. 2010] D. Balcan, B. Gonçalves, H. Hu, J. J. Ramasco, V. Colizza, and A. Vespignani, "Modeling the spatial spread of infectious diseases: the GLobal Epidemic and Mobility computational model", J. Comput. Sci. 1:3 (2010), 132-145.

[Betsch and Steinmann 2000] P. Betsch and P. Steinmann, "Conservation properties of a time FE method, I: Time-stepping schemes for N-body problems”, Int. J. Numer. Meth. Eng. 49:5 (2000), 599-638.

[Betsch and Steinmann 2001] P. Betsch and P. Steinmann, "Conservation properties of a time FE method, II: Time-stepping schemes for non-linear elastodynamics", Int. J. Numer. Meth. Eng. 50:8 (2001), 1931-1955.

[Betsch and Steinmann 2002] P. Betsch and P. Steinmann, "Conservation properties of a time FE method, III: Mechanical systems with holonomic constraints”, Int. J. Numer. Meth. Eng. 53:10 (2002), 2271-2304.

[Betsch and Steinmann 2005] P. Betsch and P. Steinmann, "Conservation properties of a time FE method, IV: Higher order energy and momentum conserving schemes", Int. J. Numer. Meth. Eng. 63:13 (2005), 1849-1897.

[Brunton et al. 2016] S. L. Brunton, J. L. Proctor, and J. N. Kutz, "Discovering governing equations from data by sparse identification of nonlinear dynamical systems", P. Natl. Acad. Sci. USA 113:15 (2016), 3932-3937.

[Diekmann et al. 2013] O. Diekmann, H. Heesterbeek, and T. Britton, Mathematical tools for understanding infectious disease dynamics, Princeton University, 2013. 
[Dirac 1950] P. A. M. Dirac, "Generalized Hamiltonian dynamics", Canadian J. Math. 2 (1950), 129-148.

[Dirac 1958] P. A. M. Dirac, "Generalized Hamiltonian dynamics”, P. Roy. Soc. A Math. Phy. 246:1246 (1958), 326-332.

[German et al. 2020] R. German, A. Djanatliev, L. Maile, P. Bazan, and H. Hackstein, "Modeling exit strategies from COVID-19 lockdown with a focus on antibody tests", preprint, 2020.

[Hethcote 2000] H. W. Hethcote, "The mathematics of infectious diseases", SIAM Rev. 42:4 (2000), 599-653.

[Ionescu et al. 2015] A. Ionescu, R. Militaru, and F. Munteanu, "Geometrical methods and numerical computations for prey-predator systems”, Brit. J. Math. Comp. Sci. 10:5 (2015), 1-15.

[Javili et al. 2019] A. Javili, A. T. McBride, and P. Steinmann, "Continuum-kinematics-inspired peridynamics: mechanical problems”, J. Mech. Phys. Solids 131 (2019), 125-146.

[Keimer and Pflug 2020] A. Keimer and L. Pflug, "Modeling infectious diseases using integrodifferential equations: optimal control strategies for policy decisions and applications in COVID19”, preprint, 2020.

[Kergaßner et al. 2020a] A. Kergaßner, C. Burkhardt, D. Lippold, M. Kergaßner, L. Pflug, D. Budday, P. Steinmann, and S. Budday, "Memory-based meso-scale modeling of Covid-19", Comput Mech. (online publication August 2020).

[Kergaßner et al. 2020b] A. Kergaßner, C. Burkhardt, D. Lippold, S. Nistler, M. Kergaßner, P. Steinmann, D. Budday, and S. Budday, "Meso-scale modeling of COVID-19 spatio-temporal outbreak dynamics in Germany", preprint, 2020.

[Kermack and McKendrick 1927] W. O. Kermack and A. G. McKendrick, "A contribution to the mathematical theory of epidemics”, P. Roy. Soc. A Math. Phy. 115:772 (1927), 700-721.

[Kermack and McKendrick 1932] W. O. Kermack and A. G. McKendrick, "Contributions to the mathematical theory of epidemics, II: The problem of endemicity", P. Roy. Soc. A Math. Phy. 138:834 (1932), 55-83.

[Kermack and McKendrick 1933] W. O. Kermack and A. G. McKendrick, "Contributions to the mathematical theory of epidemics, III: Further studies of the problem of endemicity", P. Roy. Soc. A Math. Phy. 141:843 (1933), 94-122.

[Kermack and McKendrick 1937] W. O. Kermack and A. G. McKendrick, "Contributions to the mathematical theory of epidemics, IV: Analysis of experimental epidemics of the virus disease mouse ectromelia", Epidemiol. Infect. 37:2 (1937), 172-187.

[Kermack and McKendrick 1939] W. O. Kermack and A. G. McKendrick, "Contributions to the mathematical theory of epidemics, V: Analysis of experimental epidemics of mouse-typhoid; a bacterial disease conferring incomplete immunity", Epidemiol. Infect. 39:3 (1939), 271-288.

[Levy et al. 2018] N. Levy, M. Iv, and E. Yom-Tov, "Modeling influenza-like illnesses through composite compartmental models", Phys. A 494 (2018), 288-293.

[Lew et al. 2004] A. Lew, J. E. Marsden, M. Ortiz, and M. West, "Variational time integrators", Internat. J. Numer. Methods Engrg. 60:1 (2004), 153-212.

[Linka et al. 2020] K. Linka, M. Peirlinck, F. Sahli Costabal, and E. Kuhl, "Outbreak dynamics of COVID-19 in Europe and the effect of travel restrictions", Comput. Method. Biomec. 23:11 (2020), $710-717$.

[Lu et al. 2020] H. Lu, C. W. Stratton, and Y.-W. Tang, "Outbreak of pneumonia of unknown etiology in Wuhan, China: the mystery and the miracle", J. Med. Virol. 92:4 (2020), 401-402. 
[Militaru and Munteanu 2013] R. Militaru and F. Munteanu, "Symmetries and conservation laws for biodynamical systems", Int. J. Math. Mod. Meth. Appl. Sci. 7:12 (2013), 965-972.

[Miller and Tadmor 2002] R. E. Miller and E. B. Tadmor, "The Quasicontinuum Method: overview, applications and current directions", J. Comput.-Aided Mater. 9 (2002), 203-239.

[Pastor-Satorras et al. 2015] R. Pastor-Satorras, C. Castellano, P. Van Mieghem, and A. Vespignani, "Epidemic processes in complex networks", Rev. Modern Phys. 87:3 (2015), 925-979.

[Peirlinck et al. 2020] M. Peirlinck, K. Linka, F. Sahli Costabal, and E. Kuhl, "Outbreak dynamics of COVID-19 in China and the United States", Biomech. Model. Mechan. (online publication April 2020).

[Pivovarov et al. 2018] D. Pivovarov, T. Oberleiter, K. Willner, and P. Steinmann, "Fuzzy-stochastic FEM-based homogenization framework for materials with polymorphic uncertainties in the microstructure", Internat. J. Numer. Methods Engrg. 116:9 (2018), 633-660.

[Pivovarov et al. 2019] D. Pivovarov, K. Willner, and P. Steinmann, "On spectral fuzzy-stochastic FEM for problems involving polymorphic geometrical uncertainties", Comput. Methods Appl. Mech. Engrg. 350 (2019), 432-461.

[Rahmandad and Sterman 2008] H. Rahmandad and J. Sterman, "Heterogeneity and network structure in the dynamics of diffusion: comparing agent-based and differential equation models", Manage. Sci. 54:5 (2008), 998-1014.

[Saeb et al. 2016] S. Saeb, P. Steinmann, and A. Javili, "Aspects of computational homogenization at finite deformations: a unifying review from Reuss' to Voigt's bound", Appl. Mech. Rev. 68:5 (2016), art. id. 050801.

[Seroussi et al. 2019] I. Seroussi, N. Levy, D. Paolotti, N. Sochen, and E. Yom-Tov, "On the use of multiple compartment epidemiological models to describe the dynamics of influenza in Europe", 2019. arXiv

[Yamazaki and Wang 2017] K. Yamazaki and X. Wang, "Global stability and uniform persistence of the reaction-convection-diffusion cholera epidemic model", Math. Biosci. Eng. 14:2 (2017), 559579 .

Received 7 Jun 2020. Revised 9 Jul 2020. Accepted 11 Aug 2020.

PAUL STEINMANN: paul.steinmann@fau.de

Institute of Applied Mechanics, Friedrich-Alexander Universität Erlangen-Nürnberg, Erlangen, Germany

and

Glasgow Computational Engineering Centre, University of Glasgow, Glasgow, United Kingdom 


\section{MATHEMATICS AND MECHANICS OF COMPLEX SYSTEMS}

\author{
EDITORIAL BOARD \\ ANTONIO CARCATERRA \\ ERIC A. CARLEN \\ FRANCESCO DELL'ISOLA \\ RAFFAELE ESPOSITO \\ ALbERT FANNJIANG \\ GILLES A. FRANCFORT \\ Pierangelo Marcati \\ Peter A. Markowich \\ MARTIN OSTOJA-STARZEWSKI \\ PiERRE SEPPECHER \\ David J. STEIGMANN \\ Paul Steinmann \\ PIERRE M. SuQueT \\ MANAGING EDITORS \\ MICOL AMAR \\ EMILIO BARCHIESI \\ MARTIN OsTOJA-STARZEWSKI \\ HONORARY EDITORS \\ TEODOR ATANACKOVIĆ \\ VICTOR BERDICHEVSKY \\ GuY BouchitTÉ \\ FELIX DARVE \\ CARlo Marchioro \\ ERRICO PRESUTTI \\ MARIO PULVIRENTI \\ LuCIO Russo \\ ADVISORY BOARD \\ Holm ALTENBACH \\ HARM ASKES \\ ANDREA BRAIDES \\ MAURO CARFORA \\ ERIC DARVE \\ FABRIZIO DAVÌ \\ ANNA DE MASI \\ Emmanuele DiBenedetto \\ VICTOR A. EREMEYEV \\ BERNOLD FIEDLER \\ IRENE M. GAMBA \\ PIERRE GERMAIN \\ SERgey GaVRiLyuk \\ Timothy J. Healey \\ ROBERT P. LIPTON \\ ANGELO LUONGO \\ JUAN J. MANFREDI \\ JEAN-JACQUES MARIGO \\ ANIL MISRA \\ ROBERTO NATALINI \\ Thomas J. Pence \\ ANDREY PIATNITSKI \\ Miguel A. F. SANJUAN \\ A. P. S. Selvadurai \\ MirosLaV ŠILHAVÝ \\ GEORG STADLER \\ Guido SwEERS \\ LEV TRUSKINOVSKY \\ JUAN J. L. VELÁZQUEZ \\ VINCENZO VESPRI \\ VITALY VOLPERT
}

\section{msp.org/memocs}

Università di Roma "La Sapienza", Italia

Rutgers University, USA

(CO-CHAIR) Università di Roma "La Sapienza", Italia

(TREASURER) Università dell'Aquila, Italia

University of California at Davis, USA

(CO-CHAIR) Université Paris-Nord, France

Università dell'Aquila, Italy

DAMTP Cambridge, UK, and University of Vienna, Austria

(CHAIR MANAGING EDITOR) Univ. of Illinois at Urbana-Champaign, USA

Université du Sud Toulon-Var, France

University of California at Berkeley, USA

Universität Erlangen-Nürnberg, Germany

LMA CNRS Marseille, France

Università di Roma "La Sapienza", Italia

Università degli Studi dell'Aquila, Italy

(CHAIR MANAGING EDITOR) Univ. of Illinois at Urbana-Champaign, USA

University of Novi Sad, Serbia

Wayne State University, USA

Université du Sud Toulon-Var, France

Institut Polytechnique de Grenoble, France

Università di Roma "La Sapienza", Italia

Università di Roma Tor Vergata, Italy

Università di Roma "La Sapienza", Italia

Università di Roma "Tor Vergata", Italia

Otto-von-Guericke-Universität Magdeburg, Germany

University of Sheffield, UK

Università di Roma Tor Vergata, Italia

Università di Pavia, Italia

Stanford University, USA

Università Politecnica delle Marche, Ancona (I), Italy

Università dell'Aquila, Italia

Vanderbilt University, USA

Gdansk University of Technology, Poland

Freie Universität Berlin, Germany

University of Texas at Austin, USA

Courant Institute, New York University, USA

Université Aix-Marseille, France

Cornell University, USA

Louisiana State University, USA

Università dell'Aquila, Italia

University of Pittsburgh, USA

École Polytechnique, France

University of Kansas, USA

Istituto per le Applicazioni del Calcolo "M. Picone", Italy

Michigan State University, USA

Narvik University College, Norway, Russia

Universidad Rey Juan Carlos, Madrid, Spain

McGill University, Canada

Academy of Sciences of the Czech Republic

Courant Institute, New York University, United States

Universität zu Köln, Germany

École Polytechnique, France

Bonn University, Germany

Università di Firenze, Italia

CNRS \& Université Lyon 1, France Angelo Vulpiani \& Università di Roma La Sapienza, Italia

MEMOCS (ISSN 2325-3444 electronic, 2326-7186 printed) is a journal of the International Research Center for the Mathematics and Mechanics of Complex Systems at the Università dell'Aquila, Italy.

Cover image: "Tangle" by @ John Horigan; produced using the Context Free program (contextfreeart.org).

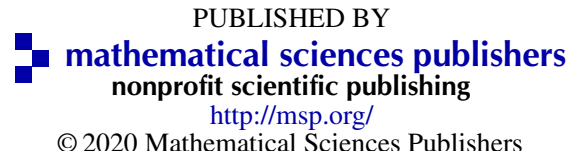

(C) 2020 Mathematical Sciences Publishers 
Mathematics and Mechanics of Complex Systems vol. 8 no. 4

On a stochastic approach to model the double phosphorylation/dephosphorylation cycle

Alberto Maria Bersani, Alessandro Borri, Francesco Carravetta,

Gabriella Mavelli and Pasquale Palumbo

A new comprehensive approach for bone remodeling under medium and high mechanical load based on cellular activity

Daniel George, Rachele Allena, Céline Bourzac, Stéphane Pallu, Morad Bensidhoum, Hugues Portier and Yves Rémond

Models for drug release of gentamicin in a polylactic acid matrix

Anna S. Morozova, Elena N. Vilchevskaya, Wolfgang H. Müller and Nikolay M. Bessonov

Analytical mechanics allows novel vistas on mathematical epidemic dynamics modeling

\section{Paul Steinmann}

A geometrically nonlinear Euler-Bernoulli beam model within strain gradient elasticity with isogeometric analysis and lattice structure applications

Loc V. Tran and Jarkko Niiranen

MEMOCS is a journal of the International Research Center for the Mathematics and Mechanics of Complex Systems at the Università dell' Aquila, Italy.

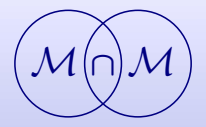

